\title{
Infecções bacterianas primárias da pele: perfil dos casos atendidos em um serviço de dermatologia na Região Amazônica, Brasil
}

Primary bacterial skin infections: profile of the cases assisted in a dermatology service in the Amazon Region, Brazil

Infecciones bacterianas primarias de piel: perfil de los casos atendidos en un servicio de dermatología en la Región Amazónica, Brasil

Carla Avelar Pires

Universidade do Estado do Pará, Belém, Pará, Brasil

Maria Amélia Lopes dos Santos

Universidade do Estado do Pará, Belém, Pará, Brasil

Bruna Feio de Oliveira

Universidade do Estado do Pará, Belém, Pará, Brasil
Camila Ribeiro de Souza

Universidade do Estado do Pará, Belém, Pará, Brasil

Larissa Nayara Martins Belarmino

Universidade do Estado do Pará, Belém, Pará, Brasil

Malu Frade Martins

Universidade do Estado do Pará, Belém, Pará, Brasil

\section{RESUMO}

As piodermites são doenças comuns, acometendo principalmente adultos e crianças. Objetivou-se neste trabalho descrever a frequência dos casos de piodermites (foliculite, erisipela, celulite, furúnculo e impetigo), o perfil epidemiológico dos pacientes acometidos por estas infecções, e observar possíveis associações com diabetes mellitus. O estudo foi realizado por meio da análise de 63 prontuários de pacientes portadores de piodermites, atendidos no período de janeiro de 2009 a outubro de 2012 no serviço de referência em dermatologia da Universidade do Estado do Pará, em Belém, Estado do Pará, Brasil. Os resultados apontaram que: as piodermites acometeram com maior frequência o gênero feminino (59\%), exceto a foliculite, que predominou no gênero masculino; em relação aos tipos de piodermites, a foliculite foi a mais frequente, com $47,61 \%$ dos casos, independente do gênero; e a faixa etária mais acometida foi de $30-39$ anos, representando $19,05 \%$ do total. Relacionando idade e gênero, a média de idade das mulheres foi de 39,4 anos e a de homens, de 30,5 anos. Dentre os casos de piodermites estudados, $8 \%$ ocorreram em pacientes diabéticos. Constatou-se que as infecções cutâneas mais prevalentes na Região Amazônica brasileira são a foliculite, o impetigo e erisipela. Não houve relação entre gênero e piodermites específicas. Um número expressivo de pacientes com as infecções bacterianas pesquisadas era de diabéticos, chamando a atenção para a necessidade de intensificar medidas preventivas contra estas infecções, principalmente neste grupo de risco.

Palavras-chave: Pioderma; Complicações do Diabetes; Infecções Bacterianas; Infecções por Bactérias Gram-Positivas.

\section{INTRODUÇÃO}

As infecções bacterianas primárias da pele acometem cerca de $7 \%$ da população, mas sua ocorrência pode variar de acordo com diversos fatores. Sabe-se que o verão predispõe as infecções cutâneas, por facilitar a instalação do calor e umidade, necessários à proliferação dos micro-organismos ${ }^{1}$. Com relação à etiopatogenia, são causadas principalmente por bactérias piogênicas dos gêneros Staphylococcus e Streptococcus ${ }^{2,3}$.

\footnotetext{
Correspondência / Correspondence / Correspondencia:

Carla Avelar Pires

Rua Bernal do Couto, $n^{\circ}$ 901, ap. 1802. Bairro: Umarizal

CEP: 66055-080 Belém-Pará-Brasil

Tel.: + 55 (91) 98826-2423

E-mail: carlaavelarpires@gmail.com
}

Além disso, sabe-se que sua frequência está relacionada a fatores ambientais; a fatores individuais, dentre os quais incluem-se aqueles relacionados à baixa resistência imunológica, como na dermatite atópica ${ }^{4}$ e diabetes mellitus; à falta de higiene; à predisposição genética; e, também, a fatores relacionados ao grau de virulência e patogenicidade do micro-organismo ${ }^{5}$, sendo também a principal complicacão de tratamentos com corticoides e imunossupressores ${ }^{6,7}$.

Em relação ao micro-organismo, o potencial infeccioso está também relacionado com a patogenicidade e o grau de virulência que decorrem, fundamentalmente, do potencial invasivo determinado pela presença de elementos antifagocitários na superfície da bactéria e da sua capacidade de produção de toxinas'. 
Sabe-se que a pele possui bactérias residentes, que vivem como comensais e transitórias que, ocasionalmente, podem colonizar a pele. Os organismos da flora residente contribuem para a resistência contra a colonização por bactérias patogênicas (Staphylococcus e Streptococcus) ao hidrolisar lipídeos e produzir ácidos graxos livres que são tóxicos para muitas bactérias. Estas bactérias, provenientes do ambiente, demonstram patogenicidade, usualmente na presença de um distúrbio de integridade da pele p $^{8}$

Aproximadamente 25-30\% dos indivíduos saudáveis apresentam colonização ocasional por S. aureus?, especialmente em axilas, períneo, faringe, mãos e narinas. A principal defesa contra os estafilococos é a fagocitose realizada pelos neutrófilos ${ }^{10}$. Entretanto, existem condições predisponentes para a colonização da pele por baixa resistência ao agente, dentre as quais estão as dos pacientes diabéticos, que são mais propensos a uma série de complicações metabólicas e infecciosas, como as alterações cutâneas bacterianas, fúngicas e virais ${ }^{11}$. Além disso, estudos anteriores demonstraram que o diabetes pode afetar algumas características biofísicas da pele ${ }^{12}$, sendo que as complicações metabólicas e alterações patológicas que ocorrem em pacientes com diabetes mellitus influenciam a ocorrência de várias dermatoses ${ }^{13,14}$. Sendo assim, a invasão direta a partir de pequenas soluções de continuidade das mucosas, da pele e seus anexos resulta em uma variedade de infecções superficiais ${ }^{1}$.

Dessa forma, considerando-se a frequência das piodermites na população, bem como sua abrangência em crianças e adultos e sua relação com fatores como o diabetes mellitus, o presente estudo tem como objetivo descrever a frequência dos casos de infecções bacterianas primárias da pele como foliculite, erisipela, celulite, furúnculo e impetigo, além de conhecer o perfil epidemiológico dos pacientes acometidos por estas piodermites em uma população da Região Amazônica e observar possíveis associações com diabetes mellitus.

\section{MATERIAIS E MÉTODOS}

Este foi um estudo transversal, retrospectivo, observacional e descritivo, com objetivo de conhecer e descrever a frequência dos casos de infecções bacterianas primárias da pele mais comuns na Região Amazônica (foliculite, erisipela, celulite, furúnculo e impetigo), além de descrever o perfil epidemiológico dos pacientes acometidos por estas infecções e observar possíveis associações destas com diabetes mellitus.

Foi utilizada uma amostra de 63 prontuários de pacientes de ambos os gêneros, todas as idades, sem distinções quanto à etnia, grau de escolaridade, níveis de renda ou estado civil, que apresentaram diagnóstico clínico das infecções supracitadas, atendidos no Ambulatório do Serviço de Dermatologia da Universidade do Estado do Pará (UEPA), uma das referências em atendimento secundário para doenças dermatológicas na região, localizado na capital, Belém, no período de janeiro de 2009 a outubro de 2012.
Foram excluídos os prontuários dos pacientes que não se encaixaram nesses requisitos. Os dados foram coletados por meio de um protocolo próprio, no qual constou: idade, gênero, residência (cidade), profissão, se o paciente tinha conhecimento de ser diabético ou não, se foi realizado exame para diabetes e o diagnóstico da infecção bacteriana.

As informações obtidas foram organizadas em um banco de dados e submetidas à estatística descritiva. Foi utilizado o software Microsoft Office Excel 2007 para a confecção das tabelas.

trabalho foi aprovado no dia 14 de dezembro de 2012 por meio de parecer consolidado pelo Comitê de Ética em Pesquisa da UEPA, Centro de Ciências Biológicas e da Saúde Campus II, sob o número CAAE 10705812.4.0000.5174, ano de 2012

\section{RESULTADOS}

De acordo com a frequência das piodermites foi observado: um caso de celulite, seis de erisipela, 30 de foliculite, quatro de furúnculo (Figura 1) e 22 casos de impetigo (Figuras 2 e 3 ). Com relação à cidade de procedência dos pacientes, eram do Município de Belém: um caso de celulite, quatro casos de erisipela, 23 casos de foliculite, quatro de furúnculo e 14 casos de impetigo; e, procedentes do interior do Estado do Pará, houve dois casos de erisipela, sete de foliculites e oito de impetigo.

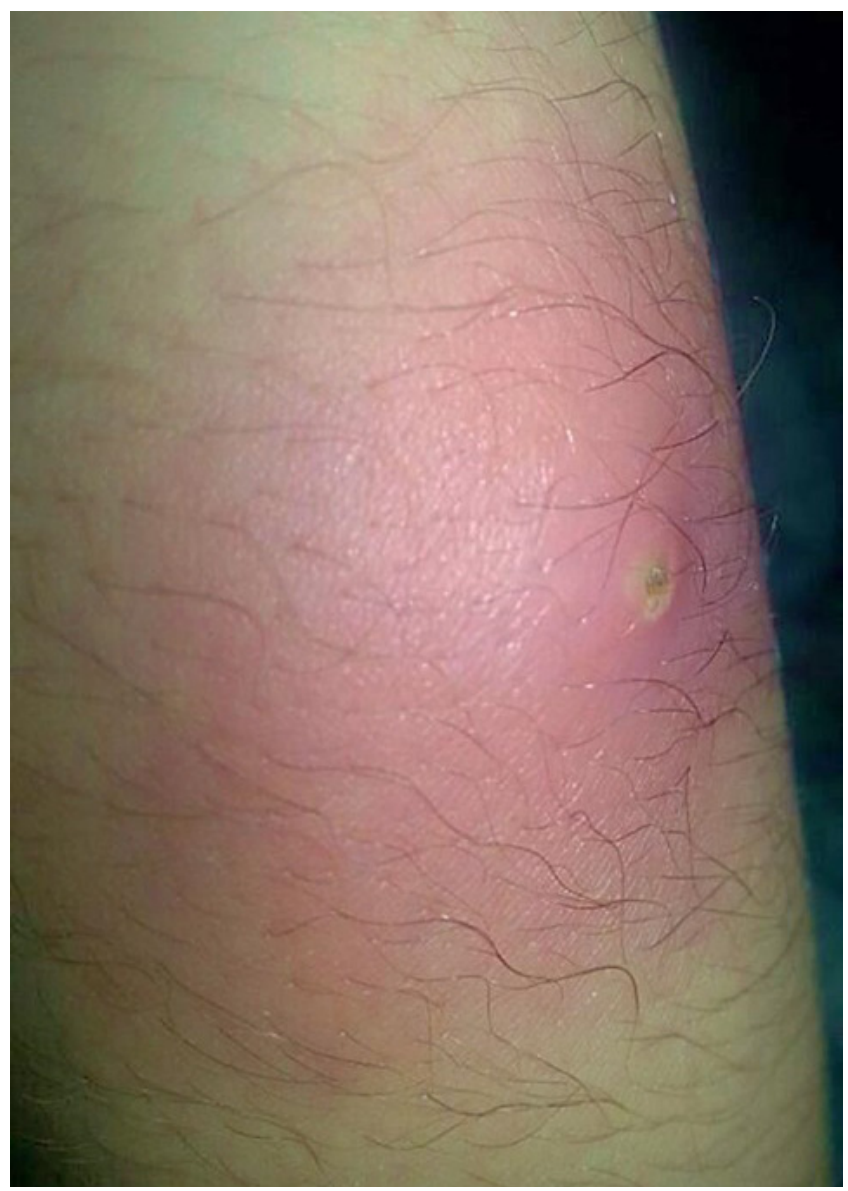

Figura 1 - Furúnculo localizado no membro inferior de paciente adulto do gênero masculino 


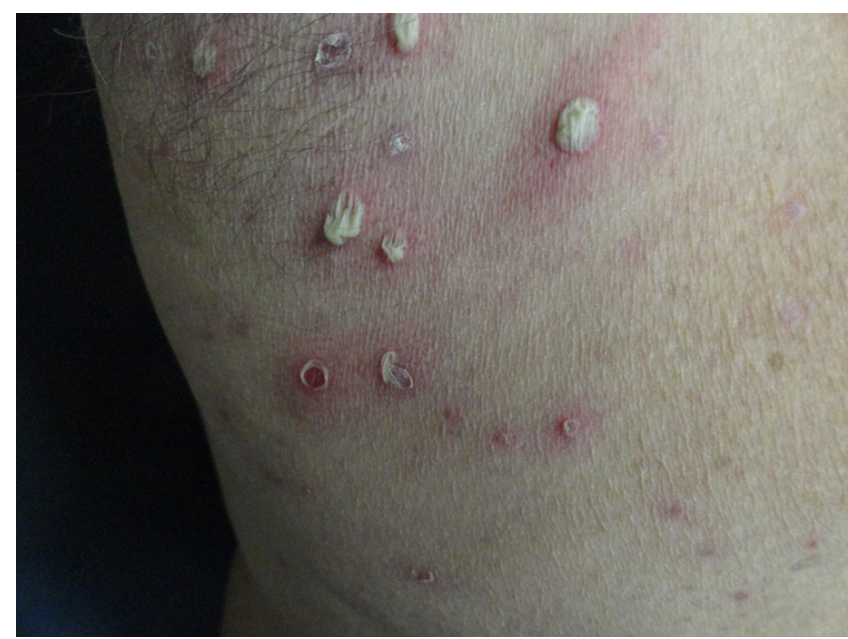

Figura 2 - Impetigo localizado em hemitórax direito de paciente adulto do gênero masculino

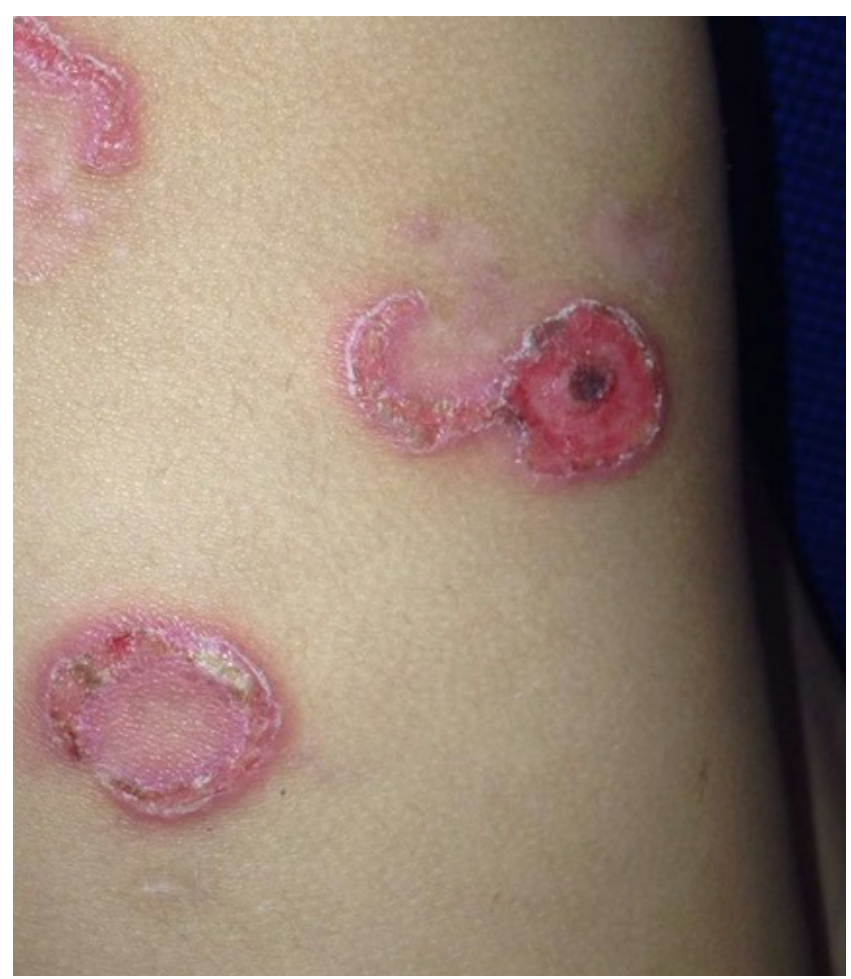

Figura 3 - Impetigo estafilocócico localizado em braço de criança do gênero feminino
De acordo com o gênero, o único caso de celulite ocorreu no gênero feminino. Dos seis casos de erisipela, dois foram em homens e quatro em mulheres. Entre os casos de foliculite, 16 aconteceram em homens e 14 em mulheres. Em relação ao furúnculo, os quatro casos foram no gênero feminino. Entre os casos de impetigo, oito ocorreram no gênero masculino e 14 no feminino.

Dentre as faixas etárias, a mais acometida pelas piodermites foi a de 30 a 45 anos incompletos, representando 28,56\% do total (Tabela 1). A média de idade das mulheres estudadas foi de 39,4 anos e a de homens, de 30,5 anos. E em relação às ocupações, dos 63 pacientes, os mais acometidos foram os estudantes (20 casos), seguidos de trabalhadores do lar (oito casos) e aposentados (sete casos).

Quanto à distribuição dos pacientes portadores de piodermites, segundo a concomitância com o diabetes, dentre os 63 pacientes pesquisados, cinco eram diabéticos e 58 não diabéticos. Sendo assim, 8\% dos pacientes eram concomitantemente diabéticos e portadores de piodermites e $92 \%$ eram portadores de piodermites sem diabetes.

Ao comparar os pacientes diabéticos e não diabéticos com as piodermites específicas dentre os cinco pacientes diabéticos, dois eram portadores de erisipela, dois de foliculite e um de furúnculo. Dentre os 58 pacientes não diabéticos, a distribuição das piodermites específica foi a seguinte: um com celulite, quatro com erisipela, $28 \mathrm{com}$ foliculite, três com furúnculo e 22 com impetigo.

A distribuição segundo a faixa etária ocorreu da seguinte forma: em pacientes com 15 anos incompletos houve 13 casos de impetigo; entre 15 e 30 anos incompletos ocorreram nove casos de foliculite, três de impetigo e um de furúnculo; na faixa etária dos 30 aos 45 anos não completos observou-se 14 pacientes com foliculite, três com impetigo e um com furúnculo; entre 45 e 60 anos incompletos, houve dois casos de foliculite, dois de impetigo, um de furúnculo e um de celulite; em pacientes com mais de 60 anos ocorreram cinco diagnósticos de foliculite, um de impetigo, seis de erisipela e um de furúnculo.

Tabela 1 - Distribuição das piodermites, de acordo com a idade, em pacientes atendidos no Ambulatório de Dermatologia da UEPA, de janeiro de 2009 a outubro de 2012, em Belém, Estado do Pará, Brasil

\begin{tabular}{|c|c|c|c|c|c|c|c|c|c|c|c|c|}
\hline \multirow{2}{*}{ Idade } & \multicolumn{2}{|c|}{ Foliculite } & \multicolumn{2}{|c|}{ Impetigo } & \multicolumn{2}{|c|}{ Erisipela } & \multicolumn{2}{|c|}{ Fúrunculo } & \multicolumn{2}{|c|}{ Celulite } & \multicolumn{2}{|c|}{ Total } \\
\hline & $N$ & $\%$ & $\mathrm{~N}$ & $\%$ & $\mathrm{~N}$ & $\%$ & $N$ & $\%$ & $N$ & $\%$ & $N$ & $\%$ \\
\hline$<15$ & - & - & 13 & 20,63 & - & - & - & - & - & - & 13 & 20,63 \\
\hline $15-30$ & 9 & 14,28 & 3 & 4,76 & - & - & 1 & 1,58 & - & - & 13 & 20,62 \\
\hline $30-45$ & 14 & 22,22 & 3 & 4,76 & - & - & 1 & 1,58 & - & - & 18 & 28,56 \\
\hline $45-60$ & 2 & 3,17 & 2 & 3,17 & - & - & 1 & 1,58 & 1 & 1,58 & 6 & 9,50 \\
\hline$>60$ & 5 & 7,93 & 1 & 1,58 & 6 & 9,52 & 1 & 1,58 & - & - & 13 & 20,61 \\
\hline Total & 30 & 47,60 & 22 & 34,90 & 6 & 9,52 & 4 & 6,32 & 1 & 1,58 & 63 & 100 \\
\hline
\end{tabular}

Sinal convencional utilizado: - Dado numérico igual a zero não resultante de arredondamento.

Fonte: Ambulatório de Dermatologia da UEPA, protocolo de pesquisa. 


\section{DISCUSSÃO}

A invasão direta por cocos gram-positivos, a partir de pequenas soluções de continuidade das mucosas, da pele e seus anexos, resulta em uma variedade de infecções superficiais ${ }^{8}$.

Dentre as infecções cutâneas mais comuns têm-se: impetigo, ectima, erisipela e foliculites ${ }^{5}$, concordando com o presente estudo no qual $58 \%$ dos casos de piodermites diagnosticadas no período estudado foram impetigo, erisipela e foliculites.

Em relação ao gênero dos pacientes, verificou-se que a porcentagem de mulheres acometidas com infecções bacterianas na pele foi maior do que a população masculina. Okajima et al ${ }^{15}$ também relatou maior incidência de algumas dermatoses como erisipela ${ }^{16}$ e celulite no gênero feminino $(54,3 \%)$, discordando de Bernardes ${ }^{17}$ que encontrou predominância no gênero masculino (55\%).

Sobre a idade dos pacientes, se considerados todos os tipos de infecção, observou-se uma grande variação, entre 1 e 90 anos, com média de idade de $35,73 \pm 23,82$ anos. $\bigcirc$ coeficiente de variação da idade foi de 66,68\%, representando, desta forma, uma casuística que engloba diversas faixas etárias. A faixa etária que obteve maior acometimento de infecção bacteriana foi a de 30 a 45 anos incompletos. Estes dados não conferem com os dados de Okajima et $a^{15}$, em que os pacientes, após a quinta década de vida, foram os mais acometidos com dermatoses bacterianas (68\%).

Quanto à distribuição de cada tipo de piodermite, de acordo com as faixas etárias, percebeu-se que entre 45 e 60 anos foi mais frequente o desenvolvimento de foliculite, enquanto nos pacientes com menos de 15 anos o maior número de casos foi de impetigo, estando de acordo com a literatura ${ }^{18}$. Uma revisão recente do Departamento de Saúde e Desenvolvimento da Criança e do Adolescente da Organização Mundial da Saúde indicou que impetigo é uma doença endêmica nesta faixa etária em muitos países tropicais e subtropicais ${ }^{19}$. No presente estudo houve maior prevalência de erisipela em idosos; assim como no de Bernardes ${ }^{17}$, que concluiu ser a faixa etária entre 60 e 70 anos a mais acometida por erisipela. Estes dados concordam também com Hadzovic-Cengic ${ }^{20}$ em que a média de idade dos pacientes com erisipela foi de 50,22 anos. Os patógenos mais comuns são Staphylococcus aureus e Streptococcus beta-hemolítico e sua presença é, em geral, de grande repercussão clínica. A erisipela é mais frequente dentre os pacientes com 50 e 70 anos de idade $^{21}$, devido a várias alterações na pele que ocorrem com o avanço da idade, além de disfunções circulatórias e pelo aumento da prevalência de infecções por determinadas bactérias.

Com relação às ocupações e piodermites, a maioria dos pacientes pesquisados eram estudantes, seguidos pelos trabalhadores do lar e aposentados, não demonstrando relação direta com nenhuma ocupação específica nesta casuística, o que pode ser observado também nos dados do Ministério da Saúde que não incluem piodermites com alguma relação direta a algum ofício $^{19}$.

Não houve diferença estatisticamente significativa ao se avaliar a relação entre a capital, o interior e as piodermites, o que corresponde aos dados na literatura. A procedência, neste estudo, está relacionada com a localização do local de atendimento, que se situa em Belém, e por isso, é desta cidade a maioria dos casos.

Ao relacionar o gênero com as doenças pesquisadas, não houve diferença estatisticamente significante entre homens e mulheres em nenhuma das afecções, o que concorda com Empinotti et $a^{5}$. Diferentemente, a pesquisa de Okajima et al ${ }^{15}$ afirma que a erisipela e a celulite são mais incidentes no gênero masculino.

Sabe-se que o diabetes mellitus é uma síndrome metabólica crônica e de origem múltipla, sendo um fator de risco para diversas complicações, entre elas algumas doenças cutâneas ${ }^{22}$ que, em sua maioria, são de etiologia infecciosa ${ }^{11}$. A neuropatia diabética e a insuficiência vascular periférica são intercorrências comuns nos pacientes diabéticos, representando fatores de risco que predispõem, também, à instalação e desenvolvimento de lesões do tegumento. $\bigcirc$ aumento da concentração da glicose no sangue do diabético provoca alterações na função leucocitária, a qual é relacionada à diminuição na difusão de nutrientes e migração dos leucócitos pelas paredes vasculares espessadas. Esses achados tornam a pele do diabético suscetível às mais variadas formas de comprometimento, principalmente por etiologia infecciosa, complicando as afecções que, geralmente, são benignas e de curta duração ${ }^{23}$. Logo, os quadros de piodermite incidem com maior gravidade nesses indivíduos ${ }^{11}$. No presente estudo, cinco dos 63 pacientes que apresentaram piodermites são diabéticos, o equivalente a $8 \%$ da amostra. Foss et $\mathrm{al}^{14}$, em uma casuística de 403 pacientes, relataram que 5\% das lesões cutâneas que acometeram os diabéticos eram as piodermites; além disso, nesta pesquisa, foi observado aumento no número de lesões cutâneas de etiologia infeciosa, tanto bacterianas como fúngicas, entre os diabéticos tipo 1 e 2 em controle metabólico inadequado. Tais dados sugerem que o descontrole metabólico do diabético proporciona maior suscetibilidade a infecções cutâneas.

\section{CONCLUSÃO}

A presente pesquisa evidenciou que as infecções bacterianas cutâneas mais prevalentes, na região do estudo, foram impetigo, erisipela e foliculite, tanto em pacientes diabéticos como não diabéticos. $\bigcirc$ gênero feminino foi $O$ mais acometido, no entanto, sem diferença estatística entre os gêneros. A faixa etária predominante foi a de 30 a 45 anos incompletos. Conclui-se ainda que um número expressivo de pacientes com as infecções bacterianas pesquisadas era de diabéticos, chamando a atenção para a necessidade de intensificar medidas preventivas contra estas infecções, principalmente neste grupo de risco. 


\title{
Primary bacterial skin infections: profile of the cases assisted in a dermatology service in the Amazon Region, Brazil
}

\begin{abstract}
Pyoderma is a common disease that occurs especially in adults and children. This study aimed to describe the frequency of pyoderma cases (folliculitis, erysipelas, cellulitis, furuncle and impetigo), the epidemiological profile of patients with these infections, and investigate possible associations with diabetes mellitus. The study was conducted by analyzing 63 patient records with pyoderma assisted between January 2009 and October 2012 in the reference service in Dermatology of the Universidade Federal do Pará, in the City of Belém, Pará State, Brazil. The results showed that: pyoderma occurs more frequently in females (59\%), except folliculitis, which predominated in males; according to the types of pyoderma, folliculitis was the most common, with $47.61 \%$ of cases, regardless of gender; and the most affected age group was 30-39 years old, representing 19.05\% of the total. Relating age and gender, the average age of women was 39.4 years old and of men, 30.5 years old. Among the case studies of pyoderma, $8 \%$ occurred in patients with diabetes. Folliculitis, impetigo and erysipelas were the most prevalent skin infections found in the Brazilian Amazon Region. There was no relationship between gender and specific pyoderma. A significant number of patients with bacterial infections was diabetic ones, pay attention to the need to enhace the preventive measures against these infections, especially in this risk group.
\end{abstract}

Keywords: Pyoderma; Diabetes Complications; Bacterial Infections; Disease Eradication.

\section{Infecciones bacterianas primarias de piel: perfil de los casos atendidos en un servicio de dermatología en la Región Amazónica, Brasil}

\section{RESUMEN}

Las piodermitis son enfermedades comunes, acometiendo principalmente a adultos y a niños. El objetivo de este trabajo fue de describir la frecuencia de los casos de piodermitis (foliculitis, erisipela, celulitis, forúnculos e empeine), el perfil epidemiológico de los pacientes acometidos por estas infecciones, y observar posibles asociaciones con diabetes mellitus. El estudio se realizó a través del análisis de 63 historiales de pacientes portadores de piodermitis, atendidos en el período de enero de 2009 a octubre de 2012 en el servicio de referencia en dermatología de la Universidad del Estado de Pará, en la Ciudad de Belém, Estado de Pará, Brasil. Los resultados señalaron que: las piodermitis acometieron con más frecuencia al género femenino (59\%), excepto la foliculitis, que predominó en el género masculino; en relación a los tipos de piodermitis, la foliculitis fue la más frecuente, con 47,61\% de los casos, independiente del género; y la franja etaria más acometida fue la de 30-39 años, representando un 19,05\% del total. Relacionando edad y género, el promedio de edad de las mujeres fue de 39,4 años y la de los hombres, de 30,5 años. Entre los casos de piodermitis estudiados, $8 \%$ ocurrió en pacientes no diabéticos. Se constató que las infecciones cutáneas más prevalentes en la Amazonia brasileña son la foliculitis, el empeine y la erisipela. No hubo relación entre género y piodermitis específicas. Un número expresivo de pacientes con las infecciones bacterianas estudiadas era de diabéticos, llamando la atención para la necesidad de intensificar medidas preventivas contra estas infecciones, principalmente en este grupo de riesgo.

Palabras clave: Pioderma; Complicaciones de la Diabetes; Infecciones Bacterianas; Erradicación de la Enfermedad.

\section{REFERÊNCIAS}

1 Pereira AL, Leal F, Azulay DR, Azulay RD. Infecções bacterianas da pele. In: Azulay RD, Azulay DR, Azylay-Abulafia L. Dermatologia. 5. ed. Rio de Janeiro: Guanabara Koogan; 2011 . p. 301-21.

2 Sampaio SAP, Rivitti EA. Dermatoses ocupacionais. In: Sampaio SAP, Rivitti EA, editores. Dermatologia. 3. ed. São Paulo: Artes Médicas; 2007. p. $1367-$ 75.

3 Pereira LB. Impetigo. An Bras Dermatolol. 2012 Sep-Oct;87(5):804.
4 Lipnharski C, d'Azevedo PA, Quinto VP, Bessa G, Bonamigo RR. Colonization by $S$. aureus increases the EASI and the number of appointments by patients with atopic dermatitis: cohort with 93 patients. An Bras Dermatol. 2013 Jul-Aug;88(4):518-21.

5 Empinotti JC, Uyeda H, Ruaro RT, Galhardo AP, Bonatto DC. Pyodermitis. An Bras Dermatol. 2012 Mar-Apr;87(2):277-84.

6 Vettorato G, Trez EG, Carvalho AVE, Garcia VD, Lecompte SM, Keitel E. Freqüência de dermatoses infecciosas em 208 pacientes transplantados renais. An Bras Dermatol. 2003 mai-jun;78(3):283-8. 
7 Petry V, Bessa GR, Poziomczyck CS, Oliveira CF, Weber MB, Bonamigo RR, et al. Bacterial skin colonization and infections in patients with atopic dermatitis. An Bras Dermatol. 2012 SepOct;87(5):729-34

8 Castro MCR, Ramos-e-Silva M. Fundamentos de dermatologia. Rio de Janeiro: Atheneu; 2009.

9 Razera F, Stefani S, Bonamigo RR, Olm GS, Dias CAG, Narvaez GA. CA-MRSA em furunculose: relato de caso do sul do Brasil. An Bras Dermatol. 2009 set-out;84(5):515-18.

10 Veronesi R. Infecções estafilocóccicas e estreptococcias. In: Veronesi R, Focaccia R. Tratado de infectologia. 3. ed. São Paulo: Atheneu; 2005. p. $861-96$.

11 Minelli L, Nonino AB, Salmazo JC, Neme L, Marcondes M. Diabetes mellitus e afecções cutâneas. An Bras Dermatol. 2003 nov-dez;78(6):735-47.

12 Seité S, Khemis A, Rougier A, Ortonne JP. Importance of treatment of skin xerosis in diabetes. J Eur Acad Dermatol Venereol. 2011 May;25(5):607-9.

13 Ragunatha S, Anitha B, Inamadar AC, Palit A, Devanmani SS. Cutaneous disorders in 500 diabetic patients attending diabetic clinic. Indian J Dermatol. 2011 Mar-Apr;56(2):160-4

14 Tiraboschi-Foss N, Silva SC, Pagnano PMG, Foss MC. Estudo das dermatopatias de uma amostra populacional de diabéticos da região de Ribeirão Preto (SP). An Bras Dermatol. 1989;64(6):31 1-5.

15 Okajima RMI, Freitas THP, Zaitz T. Estudo clínico de 35 pacientes com diagnóstico de erisipela internados no Hospital Central da Irmandade da Santa Casa de Misericórdia de São Paulo. An Bras Dermatol. 2004 mai-jun;79(3):295-303.
16 Chartier MDC, Grosshans MDE. Erysipelas. Int J Dermatol. 1990 Sep;29(7):459-67.

17 Bernardes CHA, Cardoso KT, Augusto JCA, Santos JR, Lopes LTC, Santos LM. Experiencia clínica na avaliação de 284 casos de erisipela. An Bras Dermatol. 2002 set-out;77(5):605-9.

18 Steer AC, Jenney AWJ, Kado J, Batzloff MR, Vicente $S L$, Wagatakirewa $L$, et al. High burden of impetigo and scabies in a tropical country. PLoS Negl Trop Dis. 2009 Jun;3(6):e467.

19 World Health Organization. Department of Child and Adolescent Health and Development. Epidemiology and anagement of common skin diseases in children in developing countries. Geneva: World Health Organization; 2005. 54 p.

20 Hadzovic-Cengic M, Sejtarija-Memisevic A, Koluder-Cimic N, Lukovac E, Mehanic S, Hadzic $A$, et al. Cellulitis: epidemiological and clinical characteristics. Med Arch. 2012;66 Suppl 1:51-3.

21 Dinato SLM, Oliva R, Dinato MM, Macedo-Soares A, Bernardo WM. Prevalência de dermatoses em idosos residentes em instituição de longa permanência. Rev Assoc Med Bras. 2008 novdez;54(6):544-5.

22 Sociedade Brasileira de Diabetes. Consenso Brasileiro sobre Diabetes 2002: diagnóstico e classificação do diabetes melito e tratamento do diabetes melito do tipo 2. Rio de Janeiro: Diagraphic; 2003.

23 Koivukangas V, Annala AP, Salmela PI, Oikarinen A. Delayed restoration of epidermal barrier function after suction blister injury in patients with diabetes mellitus. Diabet Med. 1999 Jul;16(7): 563-7. 